\title{
Sodium-Glucose Co-transporter 2 (SGLT2) Inhibitor: Comparing Trial Data and Real-World Use
}

\author{
Andrew McGovern (D) - Michael Feher · Neil Munro • \\ Simon de Lusignan
}

Received: February 9, 2017 / Published online: March 21, 2017

(C) The Author(s) 2017. This article is an open access publication

\begin{abstract}
Introduction: The first cardiovascular safety trial in the sodium-glucose co-transporter-2 (SGLT2) inhibitor drug class, the Empagliflozin Cardiovascular Outcomes and Mortality in Type 2 Diabetes (EMPA-REG OUTCOME) trial, demonstrated significant cardiovascular risk reduction with empagliflozin. It is currently not clear what proportions of people with type 2 diabetes (T2DM) have the same high cardiovascular risk as those included in the trial, and will therefore be likely to experience the same cardiovascular benefit. We aimed to identify and describe the proportion of people with T2DM from a
\end{abstract}

Enhanced content To view enhanced content for this article go to http://www.medengine.com/Redeem/ 89F7F060645FFEF1.

Electronic supplementary material The online version of this article (doi:10.1007/s13300-017-0254-7) contains supplementary material, which is available to authorized users.

A. McGovern $(\varangle) \cdot$ N. Munro $\cdot$ S. de Lusignan Department of Clinical and Experimental Medicine, University of Surrey, Guildford, UK e-mail: a.mcgovern@surrey.ac.uk

M. Feher

Beta Cell Centre for Diabetes, Chelsea and

Westminster Hospital, London, UK

M. Feher

Warwick Medical School, Warwick University, Coventry, UK representative English national population who have the comparable high cardiovascular risk to those included in the EMPA-REG trial.

Method: A cross-sectional analysis of cardiovascular risk in people with T2DM and a subgroup prescribed SGLT2 inhibitors. Patients were identified from the Royal College of General Practitioners Research and Surveillance Centre database. Cardiovascular risk factors were identified from electronic patient records. Results: From 1,238,909 patients at 128 GP practices, we identified 60,327 adults with T2DM (mean age 66.1 years, SD 13.9) of whom $55.6 \%$ were male. From these 1642 (2.7\%) people had been initiated on an SGLT2 inhibitor (mean age 58.1 years, SD $10.4 ; 58.8 \%$ male). In the complete T2DM group only $15.7 \%$ (95\% CI 15.5-16.0\%) had the same high cardiovascular risk as those included in the EMPA-REG trial. In those already initiated on SGLT2 inhibitors this proportion was $11.1 \%$ (95\% CI 9.8-12.4\%). Whilst the proportion was higher in the oldest age groups, in those $70+$ years old less than a quarter met the EMPA-REG trial high cardiovascular risk criteria.

Conclusions: The EMPA-REG trial results are applicable only to a small proportion of people with T2DM and a smaller proportion of those currently treated with SGLT2 inhibitors. Additional data are required to identify any cardiovascular benefit in people with lower cardiovascular risk.

Funding: AstraZeneca. 
Keywords: Cardiovascular disease; Cross sectional analysis; Empagliflozin; EMPA-REG OUTCOME; Type 2 diabetes; SGLT2

\section{INTRODUCTION}

Until recently there was little evidence for cardiovascular risk reduction with glucose-lowering therapies in type 2 diabetes (T2DM). However, within the last year the results of two trials have demonstrated substantial cardiovascular benefit with two agents: the sodium glucose co-transporter 2 (SGLT2) inhibitor empagliflozin and then glucagon-like peptide-1 (GLP-1) analogue liraglutide. These landmark diabetes trials were the Empagliflozin Cardiovascular Outcome Event Trial in Type 2 Diabetes Mellitus Patients (EMPA-REG OUTCOME) trial and the Liraglutide Effect and Action in Diabetes: Evaluation of Cardiovascular Outcome Results (LEADER) trial, respectively $[1,2]$. Whilst ground-breaking, it will take some time before the full clinical application of these trials is realized. The EMPA-REG trial was designed as a non-inferiority randomized placebo-controlled trial to investigate cardiovascular outcomes and mortality with empagliflozin in a selected group at very high risk of cardiovascular disease (Table 1). Unexpectedly, the trial demonstrated substantial cardiovascular benefit with empagliflozin in this cohort.

Sodium glucose co-transporter 2 (SGLT2) inhibitors, also known as gliflozins, improve glycemic control through inhibition of glucose reuptake by SGLT2 in the proximal tubule of the kidney $[3,4]$. This induces glucosuria which lowers serum glucose and also induces diuresis [5]. The cardiovascular risk reduction in the EMPA-REG trial was observed to occur very early, with survival curves, compared with placebo, separating almost immediately after randomization [6]. This has led some commentators to speculate that the survival benefit is achieved not from glucose lowering but due to the diuretic effect of SGLT2 inhibition as these survival curves appear similar to those for trials of diuretics in people with heart failure $[7,8]$. However, the limited data available do not support this hypothesis [9]. Alternative mechanisms have since been suggested including a reduction in cardiac preload and afterload, or shift towards myocardial tissue use of a more efficient fuel (possibly ketone bodies) [10-13]. Additional experimentation is needed, however, to explore these further. Given this uncertainty, it is unknown if the cardiovascular benefits observed in EMPA-REG can be expected in all people with T2DM or just those with a similar, high cardiovascular risk profile to those people included in the EMPA-REG trial [10].

The aims of the study were to provide a description of the cardiovascular risk profile of a large nationally representative sample of people with T2DM treated with SGLT2 inhibitors in England and compare this to the EMPA-REG trial inclusion criteria. Additionally, we compare those treated with SGLT2 inhibitors with the wider T2DM population.

\section{METHODS}

We performed a cross-sectional analysis of all people with T2DM included in the Royal College of General Practitioners Research and Surveillance Centre (RCGP RSC) database based in England, in order to identify people initiated on SGLT2 inhibitors and describe their cardiovascular risk profile. In accordance with our explicit aim to create high-quality and transparent real-world evidence $[14,15]$, we have reported the planned methods in full in the study protocol [16].

We described the proportion of people with T2DM currently using SGLT2 inhibitors who had a similar cardiovascular risk profile to those included in the EMPA-REG trial. For comparison we also reported the number of people from the complete T2DM group who had the same high cardiovascular risk. The use of English electronic health records for this purpose enabled the identification of a high-quality population denominator as all individuals are registered with a single GP practice and have a unique national identifier, the NHS number [17]. This enabled capture of an almost complete population without double counting.

At the time of this study, the RCGP RSC database contained the electronic health 
Table 1 Inclusion criteria for the EMPA-REG trial Excerpted from the supplementary material included in the EMPA-REG trial manuscript by Zinman et al. [1]

\section{Inclusion criteria}

History of myocardial infarction

History of myocardial infarction $>2$ months prior to informed consent

Coronary artery disease

Evidence of multi-vessel coronary artery disease, i.e., in $\geq 2$ major coronary arteries or the left main coronary artery, documented by any of the following:

Presence of significant stenosis: $\geq 50 \%$ luminal narrowing during angiography (coronary or multi-slice computed tomography)

Previous revascularization (percutaneous transluminal coronary angioplasty \pm stent or coronary artery bypass graft $>2$ months prior to consent

The combination of revascularization in one major coronary artery and significant stenosis ( $\geq 50 \%$ luminal narrowing) in another major coronary artery

Evidence of single-vessel coronary artery disease, $\geq 50 \%$ luminal narrowing during angiography (coronary or multi-slice computed tomography) not subsequently successfully revascularized, with at least 1 of the following:

A positive non-invasive stress test for ischemia

Hospital discharge for unstable angina $\leq 12$ months prior to consent

Unstable angina

Unstable angina $>2$ months prior to consent with evidence of single- or multi-vessel coronary artery disease

History of stroke

History of stroke (ischemic or hemorrhagic) $>2$ months prior to consent

Peripheral artery disease

Occlusive peripheral artery disease documented by any of the following:

Limb angioplasty, stenting, or bypass surgery

Limb or foot amputation due to circulatory insufficiency

Evidence of significant peripheral artery stenosis ( $>50 \%$ on angiography, or $>50 \%$ or hemodynamically significant via non-invasive methods) in 1 limb

Ankle brachial index $<0.9$ in $\geq 1$ ankle

records from 128 GP practices distributed across England (1.7\% of all practices in England). The RCGP RSC provided a sample which is representative of the UK population [18]. Included data are recorded using the Read code 5-byte version 2 coding hierarchy; this comprises comprehensive diagnosis and treatment information, prescriptions, and laboratory data [19].
We used data from all the included GP practices collated after 1 January 2016. We included all patients with a diagnosis of T2DM over 18 years old on or before this date. In those with T2DM we identified all those people initiated on SGLT2 inhibitors (canagliflozin, dapagliflozin, or empagliflozin) at any time before 1 January 2016. 
We used a two-stage informatics ontology-based process [20] to identify people with T2DM which we have reported in detail elsewhere [21]. The two stages were

1. Identification of all people with diabetes using diabetes diagnosis codes, glucose and HbA1c test results, or the use of diabetes therapies

2. Categorization by diabetes type based on their medication usage history, diabetes type codes, and other key clinical characteristics

The clinical characteristics of the whole T2DM group and subset of people using SGLT2 inhibitors were reported. These comprise age, gender, body mass index (BMI), glycated hemoglobin (HbA1c), blood pressure, diagnosis of hypertension, renal function, duration of diabetes, and current diabetes medications.

BMI was defined using the most recently recorded values. Where data on BMI were missing, we calculated BMI from the most recent weight and height measurements. HbA1c was defined using the most recent value prior to the initiation of SGLT2 inhibitor in those on SGLT2 inhibitors. Blood pressure values were taken from the most recent measurements. A diagnosis of hypertension was identified using diagnostic codes for hypertension only (not blood pressure measurements).

Renal function was reported as the proportion of people with an estimated glomerular filtration rate (eGFR) measurement of less than $60 \mathrm{ml} / \mathrm{min} / 1.73 \mathrm{~m}^{2}$. Duration of diabetes was defined as the time since the first diabetes-defining event. These events comprise first diabetes diagnostic code, first investigation result consistent with diabetes, or date of first prescription of glucose-lowering medication. We have reported the number of people with missing data for all variables.

The high cardiovascular risk inclusion criteria for the EMPA-REG study are demonstrated in Table 1. We identified people with each of these cardiovascular risk factors using clinical diagnostic codes, or other codes which identified a diagnosis of the risk factor (a complete description of these codes is provided in Supplementary Tables S1 to S6). It is possible that recording of unstable angina is limited in primary care data. As a measure of sensitivity we have also described the number of people with any recorded code for angina of any severity. We have considered a person to have the cardiovascular risk factor of interest if they have any of the disease-defining codes at any time in their clinical record.

We have reported the number of people with each risk factor and proportions with 95\% confidence intervals in those with T2DM and also in those prescribed SGLT2 inhibitors. We have also performed subgroup analyses of these proportions stratified by age group, duration of diabetes, type of SGLT2 inhibitor, and by number of current diabetes therapies. Current diabetes therapies are grouped into none, oral monotherapy, oral dual therapy, oral triple therapy (or greater), insulin therapy (any regime which involves insulin use).

\section{Compliance with Ethics Guidelines}

All data to be used was anonymized at the point of data extraction. No clinically identifiable information was made available to researchers. The study has been tested against the Health Research Authority (HRA)/Medical Research Council (MRC) "is this research" tool [22] and was considered to be an audit of current practice when compared to best available evidence. The study therefore did not require specific ethical approval. Approval for this work was granted by the RCGP RSC study approval committee. This article is based on previously conducted studies and does not involve any new studies of human or animal subjects performed by any of the authors.

\section{RESULTS}

Data were available for analysis from 1,238,909 electronic patient records at 128 general practices. From these we identified 60,327 adults with T2DM. This group was slightly older than the EMPA-REG trial cohort, with a shorter duration of diabetes, but similar mean BMI (Table 2). Within this group 1642 (2.7\%) people 
Table 2 Characteristics of with T2DM and a subgroup of people using SGLT2 inhibitors in the real world compared with those in the EMPA-REG trial

\begin{tabular}{|c|c|c|c|}
\hline Characteristic & $\begin{array}{l}\text { RCGP RSC total T2DM } \\
\text { group }(N=60,327)\end{array}$ & $\begin{array}{l}\text { RCGP RSC SGLT2 user } \\
\text { group }(N=1642)\end{array}$ & $\begin{array}{l}\text { EMPA-REG trial } \\
\text { participants }^{*}(N=4687)\end{array}$ \\
\hline Age (years) & $66.1( \pm 13.9)$ & $58.1( \pm 10.4)$ & $63.1( \pm 8.6)$ \\
\hline Male & $33,535(55.6)$ & $966(58.8)$ & $3336(71.2)$ \\
\hline \multicolumn{4}{|l|}{ Race } \\
\hline White & $42,284(70.1)$ & $1194(72.2)$ & $3403(72.6)$ \\
\hline Asian & $5706(9.5)$ & $155(9.4)$ & $1006(21.5)$ \\
\hline Black & $2648(4.4)$ & $40(2.4)$ & $237(5.1)$ \\
\hline Other & $1109(1.8)$ & $24(1.5)$ & $36(0.8)$ \\
\hline Missing & $8580(14.2)$ & $229(13.9)$ & $5(0.1)$ \\
\hline BMI $\left(\mathrm{kg} / \mathrm{m}^{2}\right)$ & $30.7( \pm 6.5)$ & $34.0( \pm 6.4)$ & $30.6( \pm 5.3)$ \\
\hline BMI not recorded & $1284(2.1)$ & $9(0.5)$ & $0(0.0)$ \\
\hline $\mathrm{SBP}(\mathrm{mmHg})$ & $132.0( \pm 14.7)$ & $130.2( \pm 13.4)$ & $135.3( \pm 16.9)$ \\
\hline $\mathrm{DBP}(\mathrm{mmHg})$ & $75.0( \pm 9.6)$ & $76.0( \pm 8.6)$ & $76.6( \pm 9.7)$ \\
\hline BP not recorded & $221(0.4)$ & $2(0.1)$ & $0(0.0)$ \\
\hline Diagnosed hypertension & $35,659(59.1)$ & $901(54.9)$ & NR \\
\hline Uncontrolled hypertension $^{\dagger}$ & $16,950(28.1)$ & $373(22.7)$ & NR \\
\hline $\mathrm{eGFR}<60 \mathrm{ml} / \mathrm{min} / 1.73 \mathrm{~m}^{2}$ & $11,167(18.5)$ & $76(4.6)$ & $0(0.0)$ \\
\hline \multicolumn{4}{|l|}{ Duration of diabetes } \\
\hline$\leq 1$ years & $5320(8.8)$ & $45(2.7)$ & $128(2.7)$ \\
\hline$>1-5$ years & $17,911(29.7)$ & $314(19.1)$ & $712(15.2)$ \\
\hline$>5-10$ years & $18,963(31.4)$ & $604(36.8)$ & $1175(25.1)$ \\
\hline$>10$ years & $18,133(30.1)$ & $679(41.4)$ & $2672(57.0)$ \\
\hline \multicolumn{4}{|l|}{ Other glucose-lowering therapy } \\
\hline Metformin & $35,146(58.3)$ & $1416(30.2)$ & $3459(73.8)$ \\
\hline Insulin & $8128(13.5)$ & $447(9.5)$ & $2252(48.0)$ \\
\hline Sulfonylurea & $14,765(24.5)$ & $800(17.1)$ & $2014(43.0)$ \\
\hline Dipeptidyl peptidase- 4 inhibitor & $7236(12.0)$ & $605(12.9)$ & $529(11.3)$ \\
\hline Thiazolidinedione & $1875(3.1)$ & $144(3.1)$ & $198(4.2)$ \\
\hline Glucagon-like peptide-1 agonist & $1983(3.3)$ & $360(7.7)$ & $126(2.7)$ \\
\hline Monotherapy & $21,284(35.3)$ & $89(5.5)$ & $1380(29.4)$ \\
\hline
\end{tabular}


Table 2 continued

\begin{tabular}{llll}
\hline Characteristic & $\begin{array}{l}\text { RCGP RSC total T2DM } \\
\text { group }(\boldsymbol{N}=\mathbf{6 0 , 3 2 7})\end{array}$ & $\begin{array}{l}\text { RCGP RSC SGLT2 user } \\
\text { group }(\boldsymbol{N}=\mathbf{1 6 4 2})\end{array}$ & $\begin{array}{l}\text { EMPA-REG trial } \\
\text { participants }^{*}(\boldsymbol{N}=4687)\end{array}$ \\
\hline Dual therapy & $11,375(18.9)$ & $448(27.5)$ & $2259(48.2)$ \\
\hline
\end{tabular}

Data are presented as $n(\%)$ or mean (SD)

$B M I$ body mass index, $D B P$ diastolic blood pressure, eGFR estimated glomerular filtration rate, $N R$ not reported, $S B P$ systolic blood pressure, $S D$ standard deviation

* Pooled empagliflozin participants (10 and $25 \mathrm{mg}$ doses)

$\dagger$ Most recent systolic blood pressure $\geq 140 \mathrm{mmHg}$

Table 3 Proportion of people with major cardiovascular risk factors amongst all those with type 2 diabetes $(N=60,327)$ and those prescribed an SGLT2 inhibitor $(N=1642)$

\begin{tabular}{|c|c|c|c|c|}
\hline \multirow[t]{2}{*}{ Cardiovascular risk factor } & \multicolumn{2}{|c|}{$\begin{array}{l}\text { RCGP RSC total T2DM } \\
\text { group }(N=60,327)\end{array}$} & \multicolumn{2}{|c|}{$\begin{array}{l}\text { RCGP RSC SGLT2 user } \\
\text { group }(N=1642)\end{array}$} \\
\hline & $\begin{array}{l}\text { Risk factor } \\
\text { present } n\left(\%^{*}\right)\end{array}$ & $\begin{array}{l}\text { 95\% confidence } \\
\text { intervals } \%^{*}\end{array}$ & $\begin{array}{l}\text { Risk factor } \\
\text { present } n\left(\%^{*}\right)\end{array}$ & $\begin{array}{l}\text { 95\% confidence } \\
\text { intervals } \%^{*}\end{array}$ \\
\hline Myocardial infarction & $2978(4.9)$ & $4.8-5.1$ & $65(4.0)$ & $3.2-4.8$ \\
\hline Coronary artery disease & $3542(5.9)$ & $5.7-6.0$ & $78(4.8)$ & $3.9-5.6$ \\
\hline Unstable angina & $528(0.9)$ & $0.8-0.9$ & $22(1.3)$ & $0.9-1.8$ \\
\hline Stroke & $2607(4.3)$ & $4.2-4.5$ & $44(2.7)$ & $2.1-3.3$ \\
\hline Peripheral artery disease & $2679(4.4)$ & $4.3-4.6$ & $56(3.4)$ & $2.7-4.1$ \\
\hline Any major cardiovascular risk factor & $9481(15.7)$ & $15.5-16.0$ & $182(11.1)$ & $9.8-12.4$ \\
\hline
\end{tabular}

* Percentage of people within group

had been initiated on an SGLT2 inhibitor. The majority of these were dapagliflozin (1123), followed by canagliflozin (422), and empagliflozin (160), including 63 people who had a prescription for two different SGLT2 inhibitors. People initiated on an SGLT2 inhibitor were younger than those in the EMPA-REG trial, had a higher BMI, and a broadly similar distribution of diabetes duration (Table 2). The mean HbA1c at SGLT2 initiation was $80.0 \mathrm{mmol} / \mathrm{mol}$ (SD 17.3). Twenty-eight people (1.7\%) had no HbA1c recorded prior to initiating an SGLT2 inhibitor.

Only a small proportion of people with T2DM had the same cardiovascular risk factors as people included in the EMPA-REG trial (Table 3). Similarly, only a small proportion of those treated with SGLT2 inhibitors had the same cardiovascular risk factors as people included in the EMPA-REG trail (Table 3). A larger proportion had angina of any severity (all people with T2DM: 4970 people; $8.2 \%$; 95\% CI 8.1-8.4\%, those treated with SGLT inhibitors: 128 people; $7.8 \%$; $95 \%$ CI $13.2-16.1 \%$ ) or any major risk factor or angina of any severity (all people with T2DM: 11,704 people; $19.4 \%$; 95\% CI 19.1-19.7\%, those treated with SGLT inhibitors: 240 people; $14.6 \%$; 95\% CI 13.2-16.1\%).

In subgroup analyses, the proportion of people with major cardiovascular risk factors increased with age but still represented a minority of people even in the oldest age groups, i.e., less than a quarter of those over 70 years old (Table 4 and Supplementary Table S7). The proportion of people with major cardiovascular risk factors varied little by number of current oral therapies (none, one, two, three or more: $14.6 \%, 14.9 \%, 14.8 \%$, and 
Table 4 Proportion of people with one or more major cardiovascular risk factor, by age group, amongst all those with type 2 diabetes $(N=60,327)$

\begin{tabular}{lllc}
\hline Age group & $\begin{array}{l}\text { Number of } \\
\text { people }\end{array}$ & $\begin{array}{l}\text { Number of people with } \\
\text { one or more major } \\
\text { cardiovascular risk factor } \boldsymbol{n}\left(\%^{*}\right)\end{array}$ & $\begin{array}{l}\text { 95\% confidence } \\
\text { interval \%* }\end{array}$ \\
\hline $18-49$ & 7526 & $225(3.0)$ & $2.7-3.3$ \\
$50-59$ & 11,145 & $988(8.9)$ & $8.4-9.3$ \\
$60-69$ & 15,643 & $2273(14.5)$ & $14.1-15.0$ \\
$70+$ & 25,971 & $5995(23.1)$ & $22.7-23.5$ \\
\hline
\end{tabular}

* Percentage of people within age group

Table 5 Proportion of people with one or more major cardiovascular risk factor, by duration of diabetes, amongst all those with type 2 diabetes $(N=60,327)$

\begin{tabular}{llll}
\hline Duration of diabetes & Number of people & $\begin{array}{l}\text { Number of people with } \\
\text { one or more major } \\
\text { cardiovascular risk factor } \boldsymbol{n}\left(\%^{*}\right)\end{array}$ & 95\% confidence interval \%* \\
\hline$\leq 1$ years & 5320 & $613(11.5)$ & $10.8-12.3$ \\
$>1-5$ years & 17,911 & $2341(13.1)$ & $12.7-13.5$ \\
$>5-10$ years & 18,963 & $2818(14.9)$ & $14.4-15.3$ \\
$>10$ years & 18,133 & $3709(20.5)$ & $20.0-21.0$ \\
\hline
\end{tabular}

* Percentage of people within diabetes duration group

$12.2 \%$, respectively) but was higher in people on insulin therapy (23.5\%) (Supplementary Table S8). The proportion of people with a major cardiovascular risk factor was higher in people with a longer duration of diabetes (Table 5) with just over 20\% prevalence in those with a history of diabetes longer than 10 years. The proportion of people with a major cardiovascular risk factor was higher in those on empagliflozin (16.3\%) than dapagliflozin $(10.2 \%)$ or canagliflozin $(12.8 \%)$, although the difference was only significant between empagliflozin and dapagliflozin (Supplementary Table S9).

\section{DISCUSSION}

In a large sample of people with T2DM in England only a small proportion (15.7\%; 95\% CI $15.5-16.0 \%)$ had the same high cardiovascular risk as those included in the EMPA-REG trial. In those already initiated on SGLT2 inhibitors the proportion was smaller still $(11.1 \%$; $95 \%$ CI $9.8-12.4 \%)$ and this was a younger group. Whilst the proportion was higher in the oldest age groups, even in those 70 years old and over, this still comprised less than a quarter. Similarly, the proportion was higher in those with the longest duration of diabetes, but only comprised just over $20 \%$ in those with a duration of diabetes longer than 10 years.

\section{Implications of the Findings}

The cardiovascular benefits identified by the EMPA-REG trial were a surprising but very welcome finding. However, it is important to be clear that the trial's participants were selected on the basis of very high cardiovascular risk. We can therefore only expect similar cardiovascular benefits in the same high risk group in the real world. Our data suggest that this makes up only a small but not insubstantial proportion of 
those with T2DM. It also suggests that current SGLT2 inhibitor use occurs in a subgroup of people with T2DM who are of a younger age and have a lower cardiovascular risk than most people with T2DM. Any cardiovascular benefit in these people is uncertain. There are also a large proportion of people with high cardiovascular risk who are not currently treated with an SGLT2 inhibitor. Therefore, there is some scope to consider extending the use of empagliflozin in people with high cardiovascular risk if clinically appropriate.

The presence of cardiovascular risk factors was found, unsurprisingly, to be highest in the oldest age groups. It is therefore likely that this group is most likely to gain the largest cardiovascular risk reduction from SGLT2 inhibitor use. However, the use of SGLT2 inhibitors in this group can be problematic as the elderly are more likely to experience volume depletion, more likely to have baseline renal impairment, and have more significant sequelae from urinary tract infections [23-27].

Other cardiovascular safety trials are still ongoing and will help to explore questions around whether these benefits are a class effect and whether they can also be seen in lower cardiovascular risk groups. The Multicenter Trial to Evaluate the Effect of Dapagliflozin on the Incidence of Cardiovascular Events (DECLARE-TIMI 58) and CANagliflozin cardioVascular Assessment Study (CANVAS) trials with dapagliflozin and canagliflozin, respectively, are the two major ongoing trials with currently approved SGLT2 inhibitors [28, 29]. Their inclusion criteria are somewhat broader than those of the EMPA-REG trial, with lower cardiovascular risk participants enrolled. Whilst these trial results are awaited, a comparison of the characteristics of people treated with T2DM in clinical practice with those of the EMPA-REG trial is important to enable an understanding of how the existing data can be applied in practice. We provide a description of the cardiovascular risk profile of a large national sample of people with T2DM treated with SGLT2 inhibitors in England and compare this to the EMPA-REG trial inclusion criteria. We also provide a comparison with the complete denominator group (all people with identified T2DM).
It should be clearly stated that SGLT2 inhibitors have benefits beyond cardiovascular risk reduction: predominantly improved glycemic control and weight loss [30]. Our real-world data demonstrate that people prescribed SGLT2 inhibitors have a higher BMI than others with T2DM. This trend suggests that the weight loss benefits of the class are a key consideration in prescribing. Whilst our analysis demonstrates that the cardiovascular benefit identified in the EMPA-REG trial can only be extrapolated to a small proportion of those currently prescribed SGLT2 inhibitors, this group will still experience glycemic and weight loss benefits.

The SGLT2 inhibitor class of medication is costlier than older therapies. The National Institute for Health and Care Excellence (NICE) in the UK has previously undertaken an economic analysis of the use of SGLT2 inhibitors and considers their use to be below the cost-effectiveness thresholds [31-33]. Otherwise analyses also suggest SGLT2 inhibitors provide cost-effective benefit [34-36]. The benefits in these analyses are based on the glucose-lowering potential and do not consider the additional cardiovascular benefits seen in the EMPA-REG trial. Although we show that the trial findings can be only applied directly to a small proportion of those with T2DM, incorporation of the additional cardiovascular risk reduction into existing economic models is still likely to improve the cost-benefit estimates.

\section{Future Research}

Our data highlight the importance of further research to identify any cardiovascular benefit from SGLT2 inhibitors in lower-risk groups which make up the majority of SGLT2 inhibitor use currently. This leads to several important clinically relevant questions: Are there any cardiovascular benefits with canagliflozin and dapagliflozin (which makes up the majority of SGLT2 inhibitor prescriptions currently)? What, if any, is the degree of cardiovascular benefit afforded in the lower cardiovascular risk groups? The ongoing cardiovascular safety trials with canagliflozin (CANVAS) and dapagliflozin (DECLARE-TIMI 58) will go some way to 
answering these questions $[28,29]$. The CANVAS trial is estimated to complete in 2017 and DECLARE-TIMI 58 in 2019. These trials should be supported by real-world analyses of cardiovascular outcomes in people currently treated with SGLT2 inhibitors.

The EMPA-REG, CANVAS, and DECLARE-TIMI 58 trials also exclude people with certain comorbidities. Many of those excluded may have had significant cardiovascular risk. Additional real-world evidence may also be needed to clarify whether the use of SGLT2 inhibitors in those with multimorbidity confers cardiovascular benefit.

\section{Comparison with Similar Studies}

Only one other similar study comparing the EMPA-REG trial inclusion criteria against a large sample of people with diabetes has been performed. The study in initial reporting, and not yet published in a complete manuscript, compared the criteria against a large registry-based population in the USA (the Diabetes Collaborative Registry) [37]. The authors used prior myocardial infarction, any coronary artery disease, any coronary revascularization, or peripheral artery disease to approximate the EMPA-REG criteria. They found that only $16 \%$ of those with T2DM met these approximate criteria. These results agree closely with those presented here. Our present study may provide a more representative sample of the general population with T2DM than this registry-based analysis. We were also able to provide a closer mapping of disease codes to the EMPA-REG inclusion criteria than was possible for this study.

\section{Strengths and Limitations}

The key strengths and limitations of the data source used for the study (RCGP RSC network of practices) have previously been described $[21,38]$ in accordance with European Federation for Medical Informatics Primary Care Informatics Working Group consensus recommendations [39]. Key strengths include the large sample size, high level of data completeness, and the broadly representative nature of the population sample. However, the RCGP RSC group of practices are a somewhat self-selecting group of GP practices with a slightly more affluent population than the UK average [38]. Despite this, given the size of the population analyzed (ca. $1.7 \%$ of the English population), we feel that these data provide a reasonable approximation of the UK population.

Identification of the EMPA-REG inclusion criteria risk factors from routine primary care data may be limited by a number of factors. Firstly, the possibility of missing data (either because of failure to record known diagnoses or failure to identify risk factors in clinical practice) may have led us to underestimate the number of people who meet these criteria. As mentioned, our previous analyses of this dataset have demonstrated a high level of data completeness, particularly in those with diabetes which somewhat mitigates this limitation $[21,38]$. Identification of specific inclusion criteria is limited by the clinical coding system used in UK primary care (Read codes) which does not have a code which mapped directly onto each EMPA-REG criterion. We have therefore used non-specific/broader clinical codes where no more specific code was available. This approach may have slightly overestimated the number of people meeting each inclusion criteria. Generally the Read coding system is an extensive system which facilitates very detailed coding of diagnoses and other code domains [19] such that this mapping mismatch is limited. Additional limitations of using routinely collected primary care data in research have also previously been described and include the loss of information recorded in the record as free text [40] which may have led to some missing cases in our data.

\section{CONCLUSIONS}

Extrapolation of the EMPA-REG trial cardiovascular benefits to people with T2DM in the real world should be performed with extreme caution. In a large nationally representative sample of people with T2DM we found that only a 
small proportion of people had the same high cardiovascular risk as those people included in the EMPA-REG trial. Additionally, an even smaller proportion of those currently treated with SGLT2 inhibitors had the same high risk. It remains to be seen whether people with T2DM, multimorbidity, and high cardiovascular risk (who were excluded from the trials) similarly benefit from treatment with SGLT2 inhibitors. Further data are also needed to identify possible cardiovascular benefit in the remaining lower cardiovascular risk group.

\section{ACKNOWLEDGEMENTS}

This publication has been supported by a grant from AstraZeneca including funding for publication charges and medical editorial assistance.

All authors had full access to all of the data in this study and take complete responsibility for the integrity of the data and accuracy of the data analysis. All named authors meet the International Committee of Medical Journal Editors (ICMJE) criteria for authorship for this manuscript, take responsibility for the integrity of the work as a whole, and have given final approval for the version to be published. The authors thank Reza Hajhosseiny, who provided medical editorial assistance on behalf of Spotlight Research Ltd. Funding for medical editorial support was provided by AstraZeneca Europe.

Andrew McGovern conceived the idea. Andrew McGovern, Michael Feher, Neil Munro, and Simon de Lusignan designed the study. Andrew McGovern undertook the data analysis. Andrew McGovern, Michael Feher, Neil Munro, and Simon de Lusignan contributed to the manuscript and approved the final draft.

Disclosures. Andrew McGovern receives research funding from Eli-Lilly and AstraZeneca. Simon de Lusignan receives research funding from Eli-Lilly, GlaxoSmithKline, Takeda, and AstraZeneca. Neil Munro has received financial support for research, speaker meetings, and consultancy from MSD, Merck, BMS, AstraZeneca, Pfizer, Novo, Eli-Lilly, and Sanofi-Aventis.
Michael Feher has received financial support for research, speaker meetings, and consultancy from MSD, Merck, BMS, AstraZeneca, Pfizer, Novo, Eli-Lilly, and Sanofi-Aventis.

Compliance with Ethics Guidelines. All data to be used was anonymized at the point of data extraction. No clinically identifiable information was made available to researchers. The study has been tested against the Health Research Authority (HRA)/Medical Research Council (MRC) "is this research" tool [22] and was considered to be an audit of current practice when compared to best available evidence. The study therefore did not require specific ethical approval. Approval for this work was granted by the RCGP RSC study approval committee. This article is based on previously conducted studies and does not involve any new studies of human or animal subjects performed by any of the authors.

Data Availability. The datasets generated during and/or analyzed during the current study are not publicly available as per the conditions of study approval by the RCGP RSC study approval committee, in order to protect patient confidentiality. The data can be made available to bona fide researchers on a caseby-case basis; please contact the corresponding author for further details.

Open Access. This article is distributed under the terms of the Creative Commons Attribution-NonCommercial 4.0 International License (http://creativecommons.org/licenses/ by-nc/4.0/), which permits any noncommercial use, distribution, and reproduction in any medium, provided you give appropriate credit to the original author(s) and the source, provide a link to the Creative Commons license, and indicate if changes were made.

\section{REFERENCES}

1. Zinman B, et al. Empagliflozin, cardiovascular outcomes, and mortality in type 2 diabetes. N Engl J Med. 2015;373(22):2117-28. 
2. Marso SP, et al. Liraglutide and cardiovascular outcomes in type 2 diabetes. $\mathrm{N}$ Engl J Med. 2016;375(4):311-22.

3. Fujita Y, Inagaki N. Renal sodium glucose cotransporter 2 inhibitors as a novel therapeutic approach to treatment of type 2 diabetes: clinical data and mechanism of action. J Diabetes Investig. 2014;5(3):265-75.

4. Rahmoune H, et al. Glucose transporters in human renal proximal tubular cells isolated from the urine of patients with non-insulin-dependent diabetes. Diabetes. 2005;54(12):3427-34.

5. Johnsson $\mathrm{K}$, et al. Osmotic diuresis with SGLT2 inhibition: analysis of events related to volume reduction in dapagliflozin clinical trials. Postgrad Med. 2016;128(4):346-55.

6. Daousi C, et al. Prevalence of obesity in type 2 diabetes in secondary care: association with cardiovascular risk factors. Postgrad Med J. 2006;82(966):280-4.

7. Sarafidis PA, Tsapas A. Empagliflozin, cardiovascular outcomes, and mortality in type 2 diabetes. N Engl J Med. 2016;374(11):1092-4.

8. Fischereder M, Schönermarck U. Empagliflozin, cardiovascular outcomes, and mortality in type 2 diabetes. N Engl J Med. 2016;374(11):1092-4.

9. Scheen AJ. Reappraisal of the diuretic effect of empagliflozin in the EMPA-REG OUTCOME trial: comparison with classic diuretics. Diabetes Metab. 2016;42(4):224-33.

10. Abdul-Ghani $\mathrm{M}$, et al. SGLT2 inhibitors and cardiovascular risk: lessons learned from the EMPA-REG OUTCOME study. Diabetes Care. 2016;39(5):717-25.

11. Ferrannini E, Mark M, Mayoux E. CV protection in the EMPA-REG OUTCOME trial: a "thrifty substrate" hypothesis. Diabetes Care. 2016;39:1108-14.

12. Mudaliar S, Alloju S, Henry RR. Can a shift in fuel energetics explain the beneficial cardiorenal outcomes in the EMPA-REG OUTCOME study? A unifying hypothesis. Diabetes Care. 2016;39(7):1115-22.

13. Abdul-Ghani $\mathrm{M}$, et al. SGLT2 inhibitors and cardiovascular risk: lessons learned from the EMPA-REG OUTCOME study. Diabetes Care. 2016;39(5):717-25.

14. de Lusignan S, Crawford L, Munro N. Creating and using real-world evidence to answer questions about clinical effectiveness. J Innov Health Inform. 2015;22(3):368-73.
15. McGovern A, Hinchliffe R, Munro N, de Lusignan S. Basing approval of drugs for type 2 diabetes on real world outcomes. BMJ. 2015;351:h5829.

16. McGovern A, et al. Sodium-glucose co-transporter-2 (SGLT2) inhibitors: comparing trial and real world use (study protocol). Diabetes Ther. 2017. doi:10. 1007/s13300-017-0229-81-9.

17. de Lusignan S, van Weel C. The use of routinely collected computer data for research in primary care: opportunities and challenges. Fam Pract. 2006;23(2):253-63.

18. UK Prospective Diabetes Study (UKPDS) Group. Intensive blood-glucose control with sulphonylureas or insulin compared with conventional treatment and risk of complications in patients with type 2 diabetes (UKPDS 33). UK Prospective Diabetes Study (UKPDS) Group. Lancet. 1998;352(9131):837-53.

19. de Lusignan S. Codes, classifications, terminologies and nomenclatures: definition, development and application in practice. Inform Prim Care. 2005;13(1):65-70.

20. Liaw ST, et al. Integrating electronic health record information to support integrated care: practical application of ontologies to improve the accuracy of diabetes disease registers. J Biomed Inform. 2014;52:364-72.

21. McGovern A, et al. Real-world evidence studies into treatment adherence, thresholds for intervention and disparities in treatment in people with type 2 diabetes in the UK. BMJ Open. 2016;6(11):e012801.

22. Medical Research Council and NHS Health Research Authority. Is my study research? 2016. http://www. hra-decisiontools.org.uk/research/. Accessed Dec 2016.

23. AstraZeneca. Farxiga (Dapagliflozin) [package insert]. Wilmington: AstraZeneca Pharmaceuticals LP; 2016.

24. Boehringer Ingelheim Pharmaceuticals Inc. Jardiance (Empagliflozin) [package insert]. Ridgefield: Eli Lilly and Company; 2016.

25. Janssen Pharmaceuticals Inc. Invokana (Canagliflozin) [package insert]. Titusville: Janssen Pharmaceuticals Inc.; 2016.

26. Cove-Smith A, Almond M. Management of urinary tract infections in the elderly. Trends Urol Gynaecol Sex Health. 2007;12(4):31-4.

27. Sehgal V, et al. Management of diabetes mellitus in the elderly with canagliflozin: a newer 
hypoglycemic drug on the horizon. J Pharmacol Pharmacother. 2014;5(4):227-31.

28. AstraZeneca. Multicenter trial to evaluate the effect of dapagliflozin on the incidence of cardiovascular events (DECLARE-TIMI58). 2012. https:// clinicaltrials.gov/ct2/show/NCT01730534. Accessed Dec 2016.

29. Janssen Research \& Development LLC. CANVASCANagliflozin cardioVascular Assessment Study (CANVAS). 2009. https://clinicaltrials.gov/ct2/ show/NCT01032629. Accessed Dec 2016.

30. Vasilakou D, et al. Sodium-glucose cotransporter 2 inhibitors for type 2 diabetes: a systematic review and meta-analysis. Ann Intern Med. 2013;159(4):262-74.

31. NICE. Dapagliflozin in combination therapy for treating type 2 diabetes. NICE technology appraisal guidance [TA288]. 2013.

32. NICE. Canagliflozin in combination therapy for treating type 2 diabetes. NICE technology appraisal guidance [TA315]. 2014.

33. NICE. Empagliflozin in combination therapy for treating type 2 diabetes. NICE technology appraisal guidance [TA336]. 2015.

34. Sabale U, et al. Cost-effectiveness of dapagliflozin (Forxiga(R)) added to metformin compared with sulfonylurea added to metformin in type 2 diabetes in the Nordic countries. Prim Care Diabetes. 2015;9(1):39-47.

35. van Haalen HG, et al. Cost effectiveness of adding dapagliflozin to insulin for the treatment of type 2 diabetes mellitus in the Netherlands. Clin Drug Investig. 2014;34(2):135-46.

36. Lopez JM, et al. Evaluating drug cost per response with SGLT2 inhibitors in patients with type 2 diabetes mellitus. Am Health Drug Benefits. 2015;8(6):309-18.

37. Arnold SV, et al. Defining the potential "real-world" impact of the EMPA-REG OUTCOME trial on improving cardiovascular outcomes: observations from the Diabetes Collaborative Registry (DCR). Diabetologia. 2016;59(S1):347.

38. Correa A, et al. Royal College of General Practitioners Research and Surveillance Centre (RCGP RSC) sentinel network: a cohort profile. BMJ Open. 2016;6(4):e011092.

39. de Lusignan S, et al. Routinely collected general practice data: goldmines for research? A report of the European Federation for Medical Informatics Primary Care Informatics Working Group (EFMI PCIWG) from MIE2006, Maastricht, the Netherlands. Inform Prim Care. 2006;14(3):203-9.

40. de Lusignan S, et al. Routinely-collected general practice data are complex, but with systematic processing can be used for quality improvement and research. Inform Prim Care. 2006;14(1):59-66. 